\title{
Cyclopean stimulation can influence sensations of self-motion in normal and stereoblind subjects
}

\author{
JEREMY M. WOLFE and RICHARD HELD \\ Massachusetts Institute of Technology, Cambridge, Massachusetts 02139
}

\begin{abstract}
Subjects experienced an illusion of self-motion when viewing the randomly patterned inner surface of a cylinder rotating about their main body axis. This sensation of rotation in a direction opposite to the direction of cylinder rotation is known as circular vection. An experiment was conducted to ascertain if the production of circular vection involved a binocular process in the visual system. Using dichoptic strobe illumination, stimuli were created that were identical monocularly but different binocularly. Groups of normal and stereoblind subjects were tested. The presence of purely binocular (cyclopean) stimulation increased the reported magnitude of vection for both groups. We conclude that a binocular process is involved in the production of circular vection and that this process retains its binocularity in stereoblind subjects.
\end{abstract}

When an observer views a randomly patterned field rotating around him, he may feel himself rotating in the opposite direction. This illusion of selfmotion is known as circular vection. Vection is but one of the effects induced by viewing large moving fields. As well as producing vection, a display rotating about the main axis of the body will generate a regular pattern of eye movements known as optokinetic nystagmus (OKN). If the field rotates around the subject's line of sight, two other phenomena may be measured. First, the subject will systematically mislocalize vertical and horizontal (tilt), and, second, his eyes will show cyclotorsional rotation in the direction of display rotation (torsion).

In previous studies (Wolfe \& Held, 1979; Wolfe, Held, \& Bauer, Note 1), we have shown that the processes producing tilt, torsion, and OKN can make use of purely binocular or cyclopean (Helmholtz, 1909/ 1924; Julesz, 1971) stimulation. Furthermore, we have shown that these results reflect the functioning of more than one binocular process in the visual system. This was demonstrated by testing stereoblind subjects who cannot use cyclopean stimuli in stereoscopic depth perception. We find that cyclopean stimuli also fail to influence the perceptual tilt effect in stereoblind subjects. However, these stimuli do influence the production of OKN and torsion. The stereoblind subjects used in these studies had histories of childhood strabismus. Their stereoblind-

The authors thank Julie Sandell for the figures, Janice Nagle for help with the manuscript, and Joseph Bauer for aid at every stage of the project. We also thank J. Atkinson and M. Cynader for useful suggestions. Terry $A$ llard provided the inspiration for the self-luminous fixation point. Optometric evaluations were done by $\mathbf{I}$. Mohindra and R. Owens. This research was supported in part by grants from NASA (NGL-22-009-308) and NIH (No. 5P30-EY02621 and No. 3-R01-EY01191). J. Wolfe was supported by a training grant (NIF-1-T31-GM0-7484). ness was probably caused by the unequal input to the two eyes resulting from the strabismus. Apparently, in these stereoblind subjects, the oculomotor processes responsible for the production of OKN and torsion retained binocularity during that period of development when the binocularity of the processes for tilt and stereopsis was lost.

Is there a binocular process involved in the production of vection? If so, what kind of process is it? Three possibilities exist: (1) It could resemble torsion and OKN in showing binocuiarity resistant to the forms of early deprivation that rendered our subjects stereoblind, (2) it could resemble tilt in showing an ability to use binocular stimuli in normal subjects but not in stereoblind subjects, or (3) it could resemble none of these and show no abililty to use binocular stimuli at all. In this study, we report that the process mediating circular vection in both normal and stereoblind subjects can make use of cyclopean stimulation. In this respect, it is similar to the processes responsible for torsion and OKN.

\section{GENERAL METHOD}

\section{Apparatus}

The subjects were seated in a 1.2-m-high and 1.1-m-diam cylinder. The subjects' field of view was restricted to the inner surface of the cylinder, consisting of a random pattern of 1.5- and .5-deg black dots on a white background. The cylinder was rotated either to the right or to the left at $26 \mathrm{deg} / \mathrm{sec}$. The subject sat $75 \mathrm{~cm}$ from the field. The sensation of self-motion was found to be maximized when a fixation target was provided just in front of the otherwise uniformly moving field. Accordingly, a 2.3-deg self-luminous spot, perched atop a thin metal pole, was placed at eye level directly in front of the field.

In these experiments, the moving field was illuminated stroboscopically. Two strobes (Grass Model PS-2 and Strobotac Type 1531-A) were used. To produce dichoptic stimuli, colored filters were placed over the strobes and colored goggles were placed over the eyes of the subject. This allowed light from each strobe to enter only one eye. Filters were red and green composites of 
Edmund Scientific filters with spectral properties described in Wolfe and Held (1979). The goggles provided a 100-deg field of view with $60 \mathrm{deg}$ of binocular overlap. The strobes were yoked electronically so that a flash from one strobe to one eye was accompanied by a flash from the other strobe to the other eye with an adjustable delay between the two flashes. Light reaching each eye was equated for luminance as measured by a selenium photocell with a spectral response similar to that of the human observer. Because the light seen by one eye was red while that seen by the other was green, the chromatic difference could have introduced a psychophysical time lag between physically simultaneous flashes (Mollon \& Krauskopf, 1973). Were this the case, a pendulum illuminated by the strobes and viewed through the goggles should show a depth illusion analogous to the "Pulfrich pendulum"' illusion. No such effect was seen.

\section{Procedure}

The technique of magnitude estimation was used to quantify vection. Subjects were seated in the cylinder with colored goggles in place as described above. Subjects were told to assign a value of 0 to the amount of self-motion felt while the drum was stationary. Next, the drum was set in motion with the room lights on. Direction of rotation was varied randomly from session to session. The subject was told to label the maximum self-motion felt during rotation uncer constant illumination as a 10 . The subjects were instructed to use values between 0 and 10 to describe the magnitude of self-motion felt during experimental trials. This technique of magnitude estimation is similar to that used by Dichgans and Brandt (1978) in many of their studies of vection.

\section{PILOT STUDY}

To determine the stroboscopic illumination conditions for the main experiment, two visually normal subjects were tested in a series of pilot experiments. As was found with tilt and torsion (Wolfe \& Held, 1979), the magnitude of vection increased as a monotonic function of strobe frequency. A strobe rate of $3 \mathrm{~Hz}$ was found to produce little or no sensation of self-motion. Fifteen hertz produced a sensation comparable to that obtained in steady illumination. The situation is analogous to that of a motion picture. The greater the number of frames per second, the more convincing the illusion of motion (here, selfmotion). As with a motion picture, no difference was seen whether one or both eyes were stimulated. Monocular conditions were obtained when the subject looked through the goggles while only one of the two strobes flashed. Binocular conditions were produced by having the two strobes flash simultaneously. In both cases, $3 \mathrm{~Hz}$ produced very little vection and $15 \mathrm{~Hz}$ produced a maximal sensation. At intermediate points, monocular and binocular conditions produced effects of comparable magnitudes.

OKN, which can be produced by a field rotating about the main body axis, was not present in these experiments as assessed by electrooculogram. The presence of the luminous fixation point suppressed OKN.

\section{MAIN EXPERIMENT}

\section{Method}

To show that cyclopean stimulation can influence the process producing vection, stimuli were used that were identical monocularly but different binocularly. These have been described in Wolfe and Held (1979). Two conditions of stroboscopic illumination were used: in and out of phase. In the in-phase condition, the two strobes flashed simultaneously at the same frequency. In the out-of-phase condition, the two strobes flashed at the same frequency but $180 \mathrm{deg}$ out of phase. In this condition, the strobes flashed in alternation and the combination of the two strobes flashed at twice the frequency of the single strobes. Monocular stimulation was the same in the in- and out-of-phase conditions. Thus, the transition from in-phase to out-of-phase introduced a purely binocular difference between the conditions.

To reiterate the comparison with a motion picture, each flash of a strobe is analogous to a single frame. In the in-phase condition, both eyes see the same sequence of frames. Thus, there is no advantage to two eyes over one. In the out-of-phase condition, however, one eye sees frames interpolated between those presented to the other eye. If the vection is produced by a binocular process, then both sets of "frames" can converge on one process, and the out-of-phase conditions should produce larger effects than would the in-phase condition at the same monocular strobe frequency. If the inputs to each eye are processed separately, the interpolation makes no difference, and in- and out-of-phase conditions should be identical.

It should be noted that this paradigm does not constitute a binocular summation experiment in the standard sense of that term (Blake \& Fox, 1973). As noted above, monocular and binocular in-phase conditions produce the same magnitude of effect, indicating that no summation of monocular inputs is occurring-as would be expected in a detection task, for example. This paradigm may be better thought of as an extended case of apparent motion produced by stimuli presented sequentially to each eye (Kolers, 1972). In the out-of-phase conditions, binocular apparent motion could be generated by the sequential flashes to each eye. In the in-phase condition, this is not possible. The addition of binocular apparent motion might be expected to increase the magnitude of vection if the vection process can use this purely binocular factor.

To ascertain if a binocular process was involved in the sensation of self-motion, the subjects were tested at a range of strobe frequencies in both in- and out-of-phase conditions. Each trial represented 1 of 14 possible stimulus configurations. As noted above, $3 \mathrm{~Hz}$ produced little or no self-motion, whereas $15 \mathrm{~Hz}$ produced the same maximum sensation felt during steady illumination. Therefore, in- and out-of-phase conditions were produced for seven monocular frequencies between 2.8 and $15 \mathrm{~Hz}$ to yield a total of 14 different stimulus configurations. On each trial, the subject rated a specific combination of phase and frequency. The subjects were asked to attend to the sensation of vection and not to base their responses on the apparent direction or velocity of the dots. A trial lasted until the subject made a response. Order of trials was random. Six trials were collected at each of the 14 combinations of phase and frequency for each subject. These 84 trials were run over the course of two or three sessions of less than $1 \mathrm{~h}$ each. For any subject, the length of the experiment depended upon the speed of response.

Subjects. Ten subjects were tested. Six had normal binocular vision and were corrected to at least $20 / 20$ acuity in both eyes during the experiment. Four subjects were stereoblind, as determined by their complete failure on standard tests of stereoscopic depth perception: Armed Forces Vision Tester, Randot, and Titmus Fly. The assessment showing that these subjects lacked all ability to use binocularly disparate stimuli for depth perception was confirmed independently by an experimenter and an optometrist. All except one stereoblind subject had approximately 20/20 acuity in each eye when run as subjects. One was correctable only to $20 / 40$ acuity in both eyes.

\section{Results}

Most subjects were highly consistent in their ratings of self-motion. Identical conditions produced very 
similar estimates from trial to trial. For most conditions, the range was no more than two to three units on the 10-point scale. This was the case in spite of the protestations of many subjects that the task was hard and their responses were arbitrary. The pattern of results was the same across both groups of subjects.

Figure 1 presents group means for normal and stereoblind subjects. It is clear from the figure that the out-of-phase conditions produce consistently larger estimates of vection than do in-phase conditions having the same monocular frequency. If the average in-phase response is paired with the out-ofphase response at each temporal frequency, we can test the hypothesis that out-of-phase values are less than or equal to in-phase values. A one-tailed t test rejects that hypothesis, with $t=3.86(p<.01)$ for
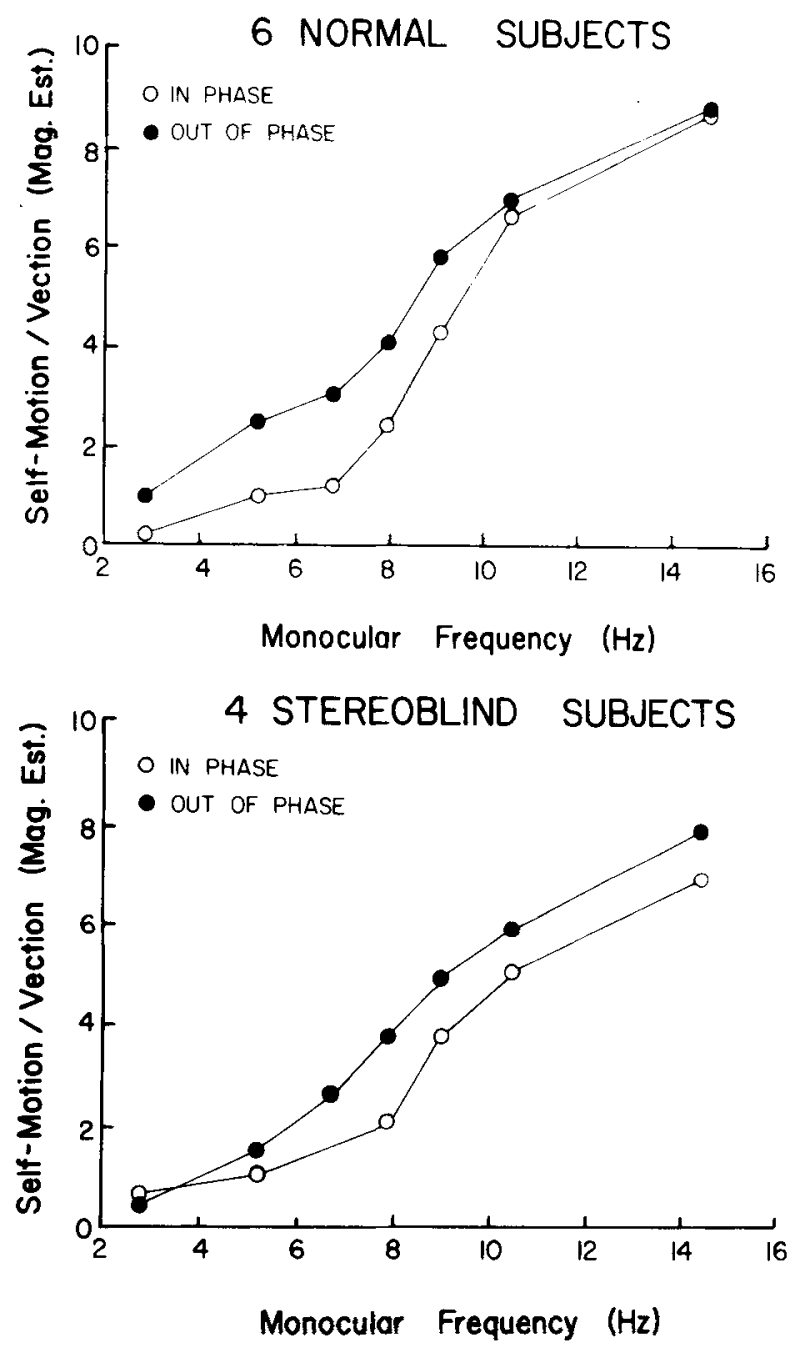

Figure 1. Magnitude of self-motion as a function of monocular strobe frequency. In-phase conditions are shown by open circles; out-of-phase, by filled circles. Top panel shows group means for six normal subjects. Bottom panel shows group means for four stereoblind subjects. Note that for both groups of subjects, out-ofphase conditions produce more vection than do in-phase conditions. This indicates that a binocular process is involved in the production of vection. normal subjects and $\mathrm{t}=3.48(\mathrm{p}<.01)$ for stereoblind subjects. No significant differences exist between the results for these two groups of subjects.

\section{DISCUSSION}

Both normal and stereoblind subjects show more vection for out-of-phase conditions than for monocularly equivalent in-phase conditions. Since the only difference between in-phase and out-of-phase conditions is in the interocular phase information, it must be concluded that the processes involved in vection are capable of using the purely binocular input. Furthermore, these processes cannot be the same as those needed for stereopsis. Stereoblind subjects, lacking the binocular process needed for stereoscopic depth perception, retain the ability to use binocular stimuli in the production of vection. This puts circular vection in the same category as eye torsion (Wolfe \& Held, 1979) and OKN (Wolfe et al., Note 1). All three phenomena are influenced by binocular input in both normal and stereoblind subjects. These results support the conclusion of Wolfe and Held (1979) that there must exist at least two binocular processes in the visual system of normal adults. One process is resistant to the effects of childhood strabismus; the other is not.

It is of interest to note that, at the start of the study, we used a fixation target that was not self-luminous and was illuminated only by the strobes. This produced inexplicably noisy results, particularly at low frequencies. The problem continued until one subject noted that, in some conditions, it was hard or impossible to keep convergence constant at the plane of the moving field. The problem seemed to be particularly severe for low-frequency out-of-phase conditions. We guessed that a continuously visible fixation point would act to stabilize convergence. Therefore, the self-luminous target was introduced in front of the field. This manipulation did eliminate problems with convergence and 'ed to the results reported above. The question of where and why vergence breaks down with intermittent stimulation remains open for future investigation.

In summary, in our dichoptic strobe paradigm, out-of-phase siimulation produced bigger estimates of self-motion than did in-phase stimulation. Inphase was identical to monocular stimulation. The only difference between in- and out-of-phase conditions was the phase shift between flashes to the two eyes, a purely binocular difference. We therefore conclude that vection is generated by a process that is capable of using purely binocular information. The fact that stereoblind subjects were also sensitive to these cyclopean stimuli indicates that the binocularity of the vection process is iesistant to the forms of developmental binncular imbalance that render people stereoblind. 


\section{REFERENCE NOTE}

1. Wolfe, J. M., Held, R., \& Bauer, J. A. A binocular contribution to the production of optokinetic nystagmus in normal and stereoblind subjects. Manuscript submitted for publication, 1980.

\section{REFERENCES}

Blake, R., \& Fox, R. The psychophysical inquiry into binocular summation. Perception \& Psychophysics, 1973, 14, 161-185.

Dichgans, J., \& BrandT, T. Visual-vestibular interaction: Effects on self-motion perception and postural control. In R. Jung (Ed.), Handbook of sensory physiology (Vol. 7). Berlin: Springer, 1978. Helmholtz, H. von. Physiological optics (Optical Society ed.).
New York: Dover, 1924. (Translated from the 3rd German ed., 1909.)

Julesz, B. Foundations of cyclopean perception. Chicago: University of Chicago Press, 1971.

Kole RS, P. A. Aspects of motion perception. Oxford: Pergamon, 1972.

Mollon, J. D., \& Krauskopf, J. Reaction time as a measure of the temporal response properties of individual colour mechanisms. Vision Research, 1973, 13, 27-40.

Wolfe, J. M., \& Held, R. Eye torsion and visual tilt are mediated by different binocular processes. Vision Research, $1979,19,917-920$.

(Received for publication January 8, 1980; revision accepted May 7, 1980.) 